
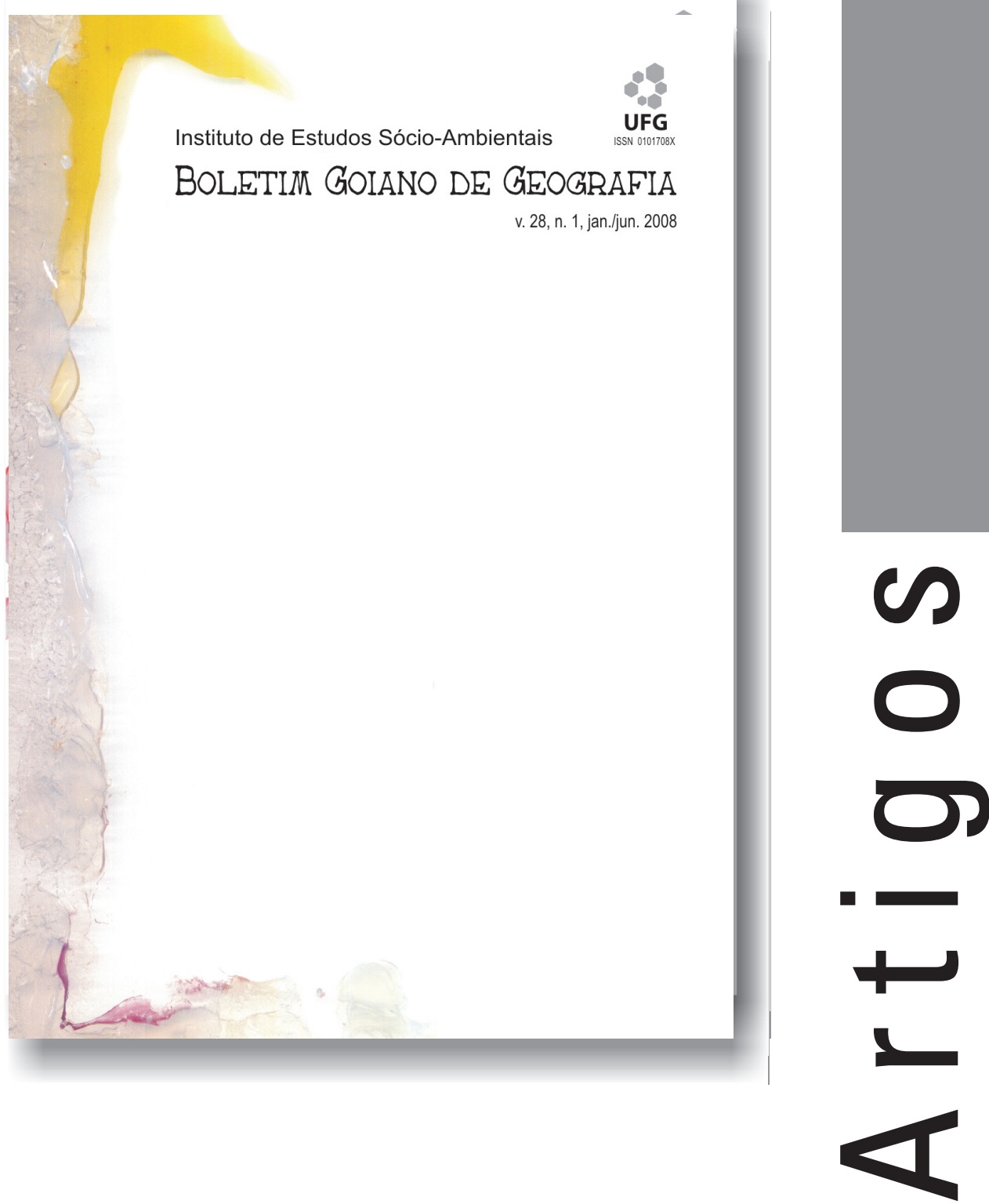


\title{
Las claves metodológicas de un proyecto aplicado al proceso de enseñanza- aprendizaje de la Geografía
}

\author{
Keys on the methodology of a project applied to the teaching-learning process in Geography \\ Alfonso García de la Vega - Universidade Autónoma de Madri \\ garcia_delavega@yahoo.es
}

\section{Resumen}

Este trabajo tiene como objeto la aplicación del proyecto geográfico como recurso didáctico generador de conocimientos significativos y de valoración crítica sobre el paisaje. Por medio del proyecto se establecen determinadas claves en el proceso de enseñanza-aprendizaje y se persigue un trabajo abierto, con especial atención a la diversidad del alumnado.

Asimismo, la interpretación y valoración del paisaje puede alcanzarse tras el reconocimiento preciso del territorio. Los conceptos se fijan a través del contacto con el entorno natural, reconociendo su problemática. De este modo, el proyecto geográfico propuesto contiene un nuevo enfoque del itinerario geográfico, que constituye el recurso didáctico medular y desde una perspectiva constructivista.

Palabras-clave: proyecto geográfico, proceso de enseñanza-aprendizaje, metodología

\begin{abstract}
This paper aims at implementing the geographical project as a didactic resource to foster the student's significant knowledge and judicious assessment on the landscape. Thereby, In this project-oriented teaching-learning process are some keys for methodology highlighted, and open work focusing on the individual's differentiation is largely pursued.

Besides, landscape interpretation and assessment can be achieved after having gained insight into the territory. The concepts are fixed in the personal contact with the natural environment and in the awareness of the issues at question. In this respect, the geographic project hereby proposed offers a new approach to the geographic itinerary, which represents a core didactic resource from a constructive view.
\end{abstract}

Key-words: geographic project, teaching-learning process, methodology 


\section{Algunas consideraciones previas}

El proyecto geográfico constituye una de las múltiples formas de contribuir al proceso de enseñanza-aprendizaje de las Ciencias Sociales en el aula y fuera de ella. Su importancia reside en que, desde el punto de vista de la didáctica, el peso de los procesos de aprendizaje se desplaza hacia los propios alumnos. De este modo, el docente se convierte en el auténtico mediador de dicho proceso, pasando a ocuparse de otros aspectos reseñables del mismo. Entre ellos se encuentran proporcionar el mayor número de recursos a los alumnos para alcanzar tal aprendizaje y atender a la diversidad del alumnado, en especial, a aquellos alumnos que precisaran necesidades educativas.

Proponemos, pues, el itinerario geográfico como el recurso medular de los objetivos del proyecto. Así, por ejemplo, el docente se interesa porque el alumno adquiera la autonomía necesaria para desplazarse por un ámbito espacial ajeno a él, desconocido. De igual manera, el alumno deberá ser capaz de descubrir y asimilar los diferentes elementos de los paisajes próximos y lejanos a su entorno. En suma, todas estas capacidades aportan al alumno diferentes tipos de recursos del conocimiento geográfico.

Precisamente, en España, en la ley educativa precedente (LOGSE, 1992) tuvo especial interés el desarrollo de los contenidos curriculares vinculados a la adquisición de los procedimientos y de las actitudes. Esto era debido a la necesidad de resaltar en el proceso de enseñanza-aprendizaje aquellos elementos opacos del currículum. En el transcurso de los años, una vez asimilados tales elementos curriculares, el interés se ha decantado hacia una política educativa común para toda la Unión Europea.

Por aquellos años, la OCDE (1990) ya había propuesto un plan de mejora de la calidad de la enseñanza, pues el acceso y la equidad en el sistema educativo europeo era un objetivo casi alcanzado. La anterior ley española hizo mayor hincapié en el factor de la equidad, en tanto que la actual Ley Orgánica de Educación (LOE, 2006) establece la calidad educativa como uno de sus principios. En su preámbulo, esta misma ley resalta la necesidad de alcanzar la convergencia educativa europea para el año 2010.

Justamente, estos objetivos pretenden desarrollar las denominadas competencias básicas, un nuevo elemento curricular que proviene de las directrices europeas para alcanzar tal convergencia (UE, Diario Oficial, L 394). Estas competencias vienen a expresar aquellas capacidades evaluables y equiparables entre los distintos estados de la Unión, que persiguen un 
“aprendizaje permanente y a lo largo de la vida”.

En definitiva, este proyecto persigue un aprendizaje más global y ajustado a la realidad, programado a través de un conjunto de tareas. De esta forma, estas tareas corresponden al "saber hacer" de las competencias básicas. Esto es, un conjunto de destrezas, habilidades y actitudes que establezcan el entramado de los aprendizajes esenciales (lectura, escritura, comprensión y expresión oral y escrita y resolución de problemas) y otros vinculados a los anteriores, que conformen los demás contenidos fundamentales del currículum, todo ello en aras de adquirir unas capacidades clave para el desarrollo del aprendizaje en la sociedad del conocimiento.

\section{Antecedentes del proyecto geográfico en la Didáctica de la Geografía}

El proyecto geográfico enlaza con un recurso habitual en la Geografía, el trabajo de campo, pero no basta con realizar una sucinta lectura de los elementos geográficos, o una explicación de las transformaciones del paisaje. El proyecto geográfico pretende plantear a los docentes una dinámica diferente y una anhelante perspectiva, donde los alumnos participen de manera activa en el proceso de enseñanza-aprendizaje. Esta intervención del alumnado se relaciona con la forma de aprender y con la dinámica de la clase, donde, como apuntábamos anteriormente, el docente es un mediador activo.

El recurso vertebrador de nuestro proyecto es el itinerario geográfico, pues permite alcanzar numerosos objetivos de forma simultánea, relacionados con la toma de decisiones, la formación de grupos, la observación y el análisis de los elementos geográficos y, por último, el establecimiento de unas hipótesis como resultado de la actividad de contrastar los resultados de la acción secular del hombre en el territorio.

La aplicación didáctica de la excursión, trabajo de campo o, en este caso, itinerario geográfico es una práctica habitual en la Didáctica de la Geografía (VIDAL BOX, 1976; GÓMEZ ORTIZ, 1986; HERRERO FABREGAT, 2001), como también en otras áreas preocupadas por los temas del medio natural. En España, estas experiencias didácticas han alcanzado su cénit con la aplicación de un Tema Transversal en el marco de la anterior Ley de Educación, que se denominó Educación Medio Ambiental (LOGSE, 1992a).

El estudio del denominado "medio", en definitiva, del territorio, desde la escuela ha sido una aportación constante desde los distintos planteamientos de los movimientos de renovación pedagógica en el transcurso del siglo 
XX (Olvera, 1987 y Carmen, 1990). En este sentido, hay que resaltar que la Institución Libre de Enseñanza fue una de las pioneras, sobre todo por la labor de Francisco Giner de los Ríos, quien probó su eficacia didáctica al aplicar la excursión al estudio de la Sierra de Guadarrama.

Tampoco hay que olvidar cuando Giner postula la vivencia del paisaje, especialmente relevante en la docencia, tanto en la dinámica de la clase y como en la atención a los alumnos.

A poco, sin embargo, que se reflexione sobre los diversos elementos en que cabe descomponer el goce que sentimos al hallarnos en medio del campo, al aire libre, se advierte que este goce no es sólo de la vista, sino que toman parte en él todos nuestros sentidos (Giner de los Ríos, Peñalara, 1915).

En torno a esta misma línea didáctica, se adscribieron otros geógrafos y naturalistas, cuyas enseñanzas en los Institutos de Enseñanza Media contemplaban el estudio del entorno natural como recurso pedagógico aglutinador de experiencias tanto personales como académicas. Este fue el caso, por ejemplo, de Dantín Cereceda, Carandell, Pericay y Vidal Box a lo largo de la primera mitad del siglo XX.

Igualmente, desde perspectivas pedagógicas muy diferentes, tanto Montesori como Freinet, propugnaron el estudio del medio natural en la formación personal del alumno fuera del aula. En suma, su práctica didáctica resultó ser beneficiosa para la enseñanza de la Geografía desde una vertiente práctica y en cualesquiera de los niveles educativos. Ahora bien, este recurso didáctico venía condicionado por las apreciaciones del maestro sobre el paisaje, reduciendo la participación activa del alumno.

Sin embargo, al tratar de desentrañar el enfoque metodológico y la batería de actividades que conforman el proyecto didáctico, parece ser primordial revisar las aportaciones previas que han contribuido a fomentar la actividad principal sobre la que giran el resto de las actividades, esto es, el itinerario geográfico. Ahora bien, más adelante mostraremos las diferencias de los itinerarios precedentes y el planteado por nosotros.

\section{Las aportaciones del proyecto al proceso de enseñanza-aprendizaje}

El proyecto geográfico es un recurso didáctico motivador, útil y valioso para el aprendizaje significativo del alumno, ya que permite el desarrollo del conocimiento, la fijación de los conceptos, desde la realidad del entorno 
natural. Al mismo tiempo conduce a elaborar una interpretación personal, generando actitudes y comportamientos sociales y éticos. Esta interpretación proporciona numerosas actividades en la atención a la diversidad del alumnado.

\section{La motivación del aprendizaje significativo en el proyecto geográfico}

La educación geográfica confluyente resulta ser aquella donde intervienen a la par el campo cognoscitivo y afectivo del alumno (BALE, 1989). Así pues, el itinerario geográfico resultaría motivador dado que el alumno tiende a establecer una relación directa con el medio natural y cultural. Además, el vínculo responde tanto a las exigencias emocionales como funcionales, puesto que enlaza con los esquemas de conocimiento previos del alumno. Esta funcionalidad del conocimiento es propugnada desde las teorías del desarrollo de un aprendizaje significativo en conexión con las estructuras cognoscitivas del alumno (COLL, 1998).

MAURI y VALLS (2004) exponen que la Geografía ayuda al alumnado a resolver aquellos problemas planteados en un espacio y un tiempo concretos, interrelacionando múltiples elementos que integran esa realidad. También resulta importante el manejo de las fuentes de información y la aplicación de determinadas técnicas de expresión gráfica y cartográfica. Además, a partir de la contribución de las disciplinas de las Ciencias Sociales, el alumno construye una visión crítica, compleja y abstracta de la realidad social.

En cuanto al grado de intervención del docente en el proceso de aprendizaje del alumno durante el itinerario geográfico se pueden fijar diversos niveles, aunque quizá el idóneo sea el de un mediador. El docente debe estimular el razonamiento de sus alumnos a partir de la observación, la clasificación y la analogía de los hechos geográficos. Ahora bien, siempre que el docente promueva nuevas situaciones de aprendizaje en la misma preparación de la salida, a través de la programación de actividades de desafío y descubrimiento.

De este modo, orientando al alumno hacia el proceso de enseñanzaaprendizaje, se fomentará en él una interpretación crítica desde la resolución, la aplicación, el análisis y la síntesis de los elementos (relieve, clima, seres vivos, suelo, agua) que integran el paisaje y en el marco del contacto directo con el paisaje a través del trabajo de campo. 
CHEVALLARD (1991) considera que la "relación didáctica” integra tanto al docente y a los alumnos como al saber. En un trabajo anterior (GARCÍA DE LA VEGA, 2004) propusimos el itinerario geográfico como un recurso generador de conocimientos, dado que suscita y permite planteamientos sobre los elementos y los hechos geográficos que parten de las ideas previas individuales del alumno y que analizadas in situ dan lugar a explicaciones más consolidadas. Además, las mismas transformaciones antrópicas constituyen un sustancioso debate sobre la pertinencia de dichos cambios y la propuesta de posibles alternativas en diferentes tipos de paisaje.

En este sentido, algunos trabajos subrayan las interrelaciones entre todos los elementos del paisaje para llevar a cabo el trabajo de campo (ALONSO OTERO, 2000). Pero también, las modificaciones en él generadas por la presencia del hombre y que, en definitiva, son las que originan cambios en el sistema natural. Descubrir este flujo de relaciones de dependencia y procesos desencadenantes de cambio es la especificidad que debe presidir el trabajo de campo.

De hecho, la LOGSE (MEC, 1992a) recogía esta interrelación que fomentaba el estudio del territorio pues las dimensiones espacial y temporal constituyen el marco de referencia idóneo donde poder entretejer contenidos, procedimientos y actitudes en el área de un estudio geográfico. Asimismo, tanto el análisis de las fuentes de información y documentación como las técnicas procedimentales en la Geografía han sido tratadas por GRAVES (1997) y revisadas por HERNÁNDEZ CARDONA (2002).

\section{La creación de valores a partir del proyecto geográfico}

El tercer aspecto propugnado como aportación pedagógica del proyecto geográfico es el significado de los hechos geográficos en el marco de los valores éticos y sociales. En tal discurso, hay que subrayar el innato componente educativo que intrínsecamente posee la Geografía, particularmente desde la docencia escolar. Perspectiva pedagógica ya reconocida en los principios de la Escuela activa de inicios del siglo XX (Institución Libre de Enseñanza, por ejemplo). 
El fundamento de esta vertiente pedagógica de la Geografía radica, ante todo, en su objeto de estudio, que persigue descubrir y explicar la interacción entre el hombre, la sociedad, y el medio donde éste se desenvuelve, el paisaje. Descubrir estas relaciones desde la asimilación de contenidos, que necesariamente supondrá el dominio de técnicas o destrezas, debe conducir necesariamente al aprendizaje o al desarrollo de una actitud crítica responsable hacia los temas medioambientales. Y que, desde la Geografía, en particular, debe suponer el cultivo de hábitos de conducta social responsable hacia nuestro entorno, pues nuestra ciencia, como las del resto del currículum, ha de contribuir, desde su particular posición, a la educación integral del alumno.

RODRÍGUEZ LESTEGÁS (2002) se expresa de similar manera para resaltar la perspectiva crítica social de la Geografía, de forma que, desde el paradigma constructivista, la Geografía supone una reconstrucción de las experiencias personales al interactuar con el medio social. Este mismo autor argumenta la importancia de llevar al aula cuestiones significativas y relevantes, propiciando la discusión y el debate. Ahora bien, esta última propuesta persigue la incorporación de los nuevos contenidos en el entramado conceptual del alumno. Su consolidación depende de la implicación activa del alumno desde una urdimbre trabada entre los cambios de carácter personal y social.

En suma, las mismas actividades señaladas más arriba sobre la estimulación del aprendizaje significativo propiciarán la asimilación de las características del paisaje, que conducirán a la elaboración personal de una actitud crítica. Si bien, esta actitud surgirá desde el conocimiento de la realidad del paisaje analizado. Precisamente, fomentar los distintos puntos de vista entre el alumnado, una vez analizada la realidad, nos conduce a establecer diferentes interpretaciones del paisaje, lo cual nos remite a un cuarto aspecto.

\section{La atención a la diversidad del alumnado en el itinerario geográfico}

Desde el final de la década de los ochenta se elaboró un programa de integración del alumnado con dificultades en el aprendizaje dentro del marco legislativo español (MARCHESI, 1990), hasta el extremo de alcanzar un amplio abanico de diagnósticos que, en cierta proporción, han sido inmersos en la enseñanza ordinaria. Se refieren a las denominadas "necesidades educativas especiales", que fueron tratadas en el desarrollo de la LOGSE (MEC, 1992c), incluyéndose nuevos perfiles a los ya existentes. No obstante, fuera 
de esos casos un tanto excepcionales, el docente tiene que tratar de atender la compleja y enriquecedora variedad del alumnado.

Justamente, O’BRIEN y GUINEY (2003) abordan la construcción del proceso enseñanza-aprendizaje, donde muchas veces existe una disparidad entre los propósitos docentes y el resultado elaborado por el alumno. Estos autores insisten en que el docente interviene decisivamente en las condiciones socioemocionales del alumno, y consideran que el docente debe atender las necesidades de aprendizaje de los diversos grupos de la clase y, excepcionalmente, aquellas necesidades específicas que demandan algunos de los alumnos.

En este mismo sentido, según COLL (1997), la actividad mental constructiva del alumno debe proporcionar la elaboración de unos esquemas de conocimiento. En consecuencia, la orientación del docente debe guiar hacia el proceso de construcción, modificación, diversificación y enriquecimiento progresivo de los esquemas de conocimiento de cada uno de los alumnos. $\mathrm{Su}$ fin es afianzar y ampliar el andamio trabado de los esquemas mentales del alumno, por medio de la asociación y dependiendo de la funcionalidad de cada uno de ellos.

En resumen, se trata de comprobar cómo esta propuesta didáctica del área de Geografía propicia la atención a la diversidad en el aula. Precisamente, el proyecto promueve tanto las relaciones entre los propios alumnos como con el adulto. En el primer caso, porque los alumnos han de formar grupos para organizar el trabajo propuesto. Además debe establecerse una dinámica de grupos flexible a lo largo del proyecto y, especialmente, en el desarrollo y secuencia temporal del itinerario geográfico, pues es la actividad medular del mismo. En el segundo caso, el docente debe saber establecer una relación que resida en la confianza con el alumno, a la vez que reafirme su seguridad y autonomía en el momento de establecer sus hipótesis de trabajo y de alcanzar unas conclusiones.

\section{El itinerario geográfico como recurso didáctico en el proyecto educativo}

Nunca el desarrollo de un itinerario geográfico ha de suponer una actividad añadida al programa de la disciplina, sino que debe interpretarse como complemento de ella y con entidad propia (GÓMEZ ORTIZ, 1986). La realización de todo itinerario geográfico ha de sustentarse en unos contenidos previos acerca del tema de estudio. Éstos deberán haber sido presentados y asimilados en el aula, pues a partir de ellos el alumno podrá establecer relaciones con todo aquello que encuentren en la realidad. 
Ahora bien, en el presente trabajo sugerimos algunas propuestas de aquel itinerario geográfico en cuanto proyecto educativo sobre la valoración del paisaje como contenido estructurador de dimensión curricular transversal. En vez de desarrollar un itinerario concreto, hemos tratado de formular las características de un prototipo de proyecto geográfico. Para ello, hemos marcado unos criterios referenciales:

- La elección de un lugar singular y significativo por cuanto que confluyen dos tipos de contenidos propios de la Geografía: el paisaje natural y las modificaciones antrópicas del mismo. Se trata de afrontar el conocimiento de los diversos usos y aprovechamientos que durante siglos ha configurado este territorio. De esta manera, adentrarse en el descubrimiento de los condicionantes naturales y circunstancias económico-sociales que se han ido sucediendo hasta llegar al actual paisaje constituye el marco del discurso a abordar.

- $\quad$ El tema nuclear tiene que transmitir suficiente controversia para generar una discusión final y propiciar unas técnicas de dinámica de grupo, ligadas a defender determinados roles con el fin de conocer todos los puntos de vista. En concreto, se trata de desarrollar el espíritu crítico de los excesos y de los perjuicios del hombre para con la naturaleza. Asimismo, se pretende forjar una determinada actitud desde el conocimiento y la propia experiencia vivida en el terreno, no obstante, sin caer en posturas demagógicas, sino tratando de abrir una vía hacia la tolerancia y la comprensión del desarrollo humano.

- El lugar elegido debe favorecer el desarrollo de las habilidades y destrezas propias de las técnicas de la Geografía. En este sentido, nos referimos a fórmulas que engloban desde realizar pequeños esbozos y figuras del paisaje, cortes topográficos -incluso geológicos, si los dominaran-, hasta utilizar las técnicas de la encuesta y de la entrevista.

\section{Observaciones de implantación educativa. Etapas y secuencia temporal}

Los requisitos educativos adecuados al proyecto geográfico podrían implantarse en todos los niveles educativos. Sin embargo, las experiencias realizadas han visto consolidados sus resultados a partir del último ciclo de 
la Etapa Primaria en adelante, puesto que los alumnos de este ciclo están más capacitados para desarrollar tal actitud crítica, siempre en función de la naturaleza y profundidad de los contenidos y objetivos a tratar. Y, por supuesto, se puede llevar a cabo en la etapa Secundaria Obligatoria y Bachillerato. Afrontar estas metas requiere, como antes se indicaba, un trabajo previo de aula enmarcado en el desarrollo curricular.

En el planteamiento del proyecto se establecen tres fases en la secuencia temporal para adaptar a la misma las técnicas de aprendizaje. Estos tres momentos constituyen la preparación en el aula, el desarrollo en el sector de estudio y, por último, la finalización o síntesis llevada a cabo de nuevo en el aula. En primer lugar, la fase de preparación se desarrollará en el aula y consistirá en la localización, clasificación, selección y archivo de información relativa a los objetivos y al contenido del itinerario geográfico. Todo ello supondrá establecer unos criterios de jerarquización y catalogación de la información recabada, criterios que deben asumir los propios alumnos. Esta tarea implica manejar distintos tipos de fuentes documentales, sea Referências, fotográfica y cartográfica, tanto histórica como actual. Todos los datos obtenidos, válidos para el análisis y su posterior interpretación del paisaje objeto de estudio, han de permitir a los alumnos formular conjeturas sobre la realidad física y humana del territorio. A esta fase se destinarán al menos dos semanas con el fin de permitir la adquisición de los conocimientos por parte del grupo de trabajo y, tras la compilación y clasificación del material, de fomentar razonamientos y elaborar estrategias.

En segundo lugar, la fase del trabajo de campo significa introducir al alumno en la lectura geográfica del paisaje. Si en la fase anterior el docente debe marcar pautas y ritmos, ahora debe orientar y dirigir, pues se trata de analizar y recomponer elementos interactuantes del paisaje. Si bien, algunos alumnos pueden estar habituados a tales ejercicios, para la mayoría resultará una novedad atractiva pero no fácil. En definitiva, se trata de anotar determinados datos actuales del paisaje y contrastarlos con la información ya disponible, pues todo ello, de vuelta al aula, les será de valiosa ayuda para profundizar en los cambios habidos en los usos del suelo.

La temporalización de la jornada de campo se establece en dos sesiones. La sesión matutina se destina a situar y conocer el ámbito geográfico en toda su dimensión, realizando algunas paradas con el fin de aproximarse al ámbito de estudio y reconocer sus elementos biofísicos. La sesión de tarde pretende centrar la atención de los alumnos en las cuestiones concretas relacionadas con el objeto de estudio del proyecto, la polémica que se pretende establecer (un embalse, una red eléctrica, un campo fotovoltaico, una urbanización, etc.). 
Por último, la fase de síntesis se desarrolla de nuevo en el aula, una vez asumido el trabajo de campo. Toda la información recabada por medio de las distintas técnicas implementadas, ya sea de toma de contacto con los hechos geográficos en el aula, o de contraste y verificación de los mismos en el propio sector de estudio durante el trabajo de campo, se retoma para su nuevo análisis y contraste. Ahora lo que interesa es la verificación de las distintas hipótesis formuladas por los distintos grupos de alumnos, así como la extracción de algunas conclusiones conforme al planteamiento esbozado al inicio del proyecto, para la redacción final del informe y su posterior exposición.

\section{Principales objetivos del proyecto}

A continuación vamos a enumerar algunos objetivos que, fundamentalmente, persiguen dos ámbitos de desarrollo en el área de la Geografía vinculado al nivel educativo correspondiente. Por una parte, desarrollar las habilidades y destrezas relacionadas con las técnicas de la Geografía. Por otra parte, propiciar una actitud crítica ante la realidad como consecuencia de un conocimiento del territorio.

Las habilidades y destrezas tienen como principal objetivo el desarrollo de la identificación de los elementos geográficos a través de las técnicas propias del área y, si fuera necesario, de otras. Entre ellas conviene resaltar, en primer lugar, la obtención y consulta de documentos diversos para la obtención de una determinada información relevante sobre hechos y fenómenos previamente delimitados del territorio objeto de examen. En este sentido, el dominio de las fuentes Referências, cartográficas y urlgráficas resulta esencial en el desarrollo del proyecto. Nuevamente, el papel mediador del docente y su conocimiento de dichas fuentes puede converger en una diversidad de resultados. Por ello, resulta conveniente que los proyectos se llevan a cabo por varios docentes de un mismo centro o de centros diferentes.

En segundo término, hay técnicas geográficas esenciales que deben ejercitarse en el trabajo previo a la salida al campo. En este caso se hallan la lectura del mapa topográfico, auxiliado con la fotografía aérea de detalle o, en todo caso, el ortofotomapa a gran escala, así como el levantamiento de los croquis panorámicos y la recogida de muestras para su posterior análisis a través de las claves de clasificación. Asimismo, y ya en el trabajo de campo, resulta muy interesante la práctica de la encuesta o entrevista a los lugareños, sobre todo para la obtención directa de datos relativos a la información del territorio. 
Por último, en tercer lugar, el establecimiento de las posibles hipótesis y conjeturas que después deberán cotejarse y valorarse con las observaciones en el campo. Una vez ordenadas las ideas y experiencias asimiladas a lo largo del desarrollo del itinerario geográfico, éstas deberán debatirse y contrastar las hipótesis que pudieron plantearse. En todo caso, dependiendo de los niveles educativos, así habremos de ajustar el tipo de técnicas que los alumnos deben iniciar, desarrollar y adquirir.

El fomento de la actitud crítica y la valoración pertinente a los resultados obtenidos provienen, por una parte, de los conocimientos adquiridos en el itinerario geográfico y, por otra, a través de las técnicas geográficas desarrolladas en la clase con el alumnado. Sin embargo, en el siguiente apartado se establecen algunas pautas para fomentar y dinamizar el trabajo en grupo, eje medular tanto de las tareas previas al itinerario como posteriores al mismo. En definitiva se trata de promover un compromiso ético de respeto hacia el entorno y, en último caso, si procede resaltar las actuaciones personales que cada uno puede elegir y llevar a efecto para responder a tal compromiso.

\section{Organización de aula: dinámica, técnicas y materiales}

En cuanto a la dinámica de trabajo del grupo-clase, el docente debe propiciar en todo momento las sesiones participativas. Parece ser que la creación de equipos de trabajo operativos en los que la transferencia de información entre sus componentes sea fluida es una manera interactiva del enriquecimiento individual y grupal de los alumnos y que tiende a retroalimentarse (Coll, 1997). Este esquema de agrupación podría rotar para hacerse permeable a las diferentes actividades propuestas, además de mejorar la dinámica grupal. Sin embargo, será conveniente que el trabajo final, esto es, el informe documental sea realizado por el equipo de trabajo establecido al inicio.

En lo relativo a la agrupación y a la secuenciación del tiempo, de vuelta en el aula, las primeras sesiones se dedicarán a la puesta en común de las observaciones y al ordenamiento y clasificación de los datos recogidos. Tras la exposición de los datos recabados por los diferentes grupos de trabajo, se dedicará una sesión a debatir las distintas posturas para alcanzar, entre todos, una valoración común sobre la incidencia física y socio-económica en el paisaje del tema común elegido en el proyecto. 


\section{Consideraciones finales}

El proyecto geográfico resulta ser un adecuado recurso didáctico para consolidar los nuevos conocimientos fuera del aula. Desde luego, reafirma la asimilación y elaboración de otros contenidos desde el paradigma constructivista. En este sentido, todos los alumnos pueden adecuar unos conocimientos mínimos desde los cuales establecer sus propias asociaciones mentales. En definitiva, se trata de fortalecer la construcción de unos aprendizajes significativos desde el conocimiento de la realidad.

Esto supone elaborar un conocimiento que conduzca a la obtención de unos argumentos que sustenten una actitud crítica consecuente. En el proyecto se establece un recurso didáctico canalizador de todo el trabajo, el itinerario geográfico. En este sentido, según hemos tratado de expresar, el itinerario es uno de los recursos más completos y, si cabe, complejos, que puede utilizarse para la docencia la Geografía. Asimismo, el itinerario exige una preparación previa del trabajo de campo así como la consolidación de los aprendizajes obtenidos.

Por todo ello, el enfoque de este proyecto de Geografía propone obtener diversas estrategias mentales entre el alumnado desde la trabazón de los conceptos adquiridos, pues la labor del docente en este tipo de recursos didácticos debe inducir a la exploración y al aprendizaje, tanto individual como colectivo, fomentando un ambiente de generosa complicidad en el descubrimiento. Su fin es el análisis del paisaje desde los datos obtenidos en el itinerario geográfico y la interpretación de las múltiples facetas de la realidad, hasta el extremo posible de alcanzar compromisos y actitudes reales ante la sociedad y la naturaleza.

En conclusión, el docente pretende desarrollar las habilidades y destrezas del área tanto como alcanzar una actitud crítica en el alumnado. Al tiempo, la dinamización y la organización de la clase, así como la atención a la diversidad, son algunos ejes capitales del trabajo a desarrollar por el docente en su tarea educativa.

\section{Bibliografía}

ALONSO OTERO, F. “Trabajo de campo: visita al Cañón del río Lobos (Soria)”, pp. 357-368 en MARTÍNEZ DE PISÓN, E. (dir.): Estudios sobre el paisaje, Universidad Autónoma de Madrid y Fundación de los Duques de Soria, Colección Estudios nº 67, 2000. 
BALE, J. Didáctica de la Geografía en la escuela primaria, Ediciones Morata y Ministerio de Educación y Ciencia, Madrid, 1989.

CARMEN, L. del. Investigación del medio y aprendizaje, Graó, Barcelona, 1990.

CHEVALLARD, Y. La transposición didáctica, Aique, Buenos Aires, 1991.

COLL, C. Aprendizaje escolar y construcción del conocimiento, Paidós, Barcelona, 1997.

COLL, C. "La teoría genética y los procesos de construcción del conocimiento en el aula", pp. 15-52 en VV.AA.: Piaget en la educación. Debate en torno de sus aportaciones, Paidós, México, 1998.

GARCÍA DE LA VEGA, A. "El itinerario geográfico como recurso didáctico para la valoración

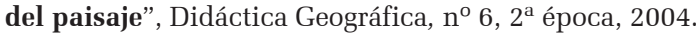

GÓMEZ ORTIZ, A. "Los itinerarios pedagógicos como recurso didáctico en la enseñanza de la Geografía en la EGB”, Didáctica Geográfica, nº 14, 1986.

GRAVES, N. J. La enseñanza de la Geografía, Visor, $2^{\text {a }}$ edición ampliada, Madrid, (1 $1^{\text {a }}$ edición 1985). 1997.

GUREVICH, R. Conceptos y problemas en Geografía, pp. 159-182 en AISENBERG, B. y ALDEROQUI, S. (comp.): Didáctica de las ciencias sociales II, Paidós, Buenos Aires, 1998.

HERNÁNDEZ CARDONA, F. X. Didáctica de las ciencias sociales, geografía e historia, Graó, Barcelona, 2002.

HERRERO FABREGAT, C. La Sierra Norte de Madrid. El Berrueco, Torrelaguna, Patones y El Atazar. Comunidad Autónoma de Madrid, 2001.

LÓPEZ MARTÍNEZ, J. "Las competencias básicas del curriculo en la LOE”, Congreso Internacional "Educación y Sociedad”, Granada, http://congreso. codoli.org/ conferencias/ JuanLopez.pdf. 2006.

MARCHESI, A. "Del lenguaje de las deficiencias a las escuelas inclusivas” pp. 21-43, en MARCHESI, A.; COLL, C. y PALACIOS, J. (comp.): Desarrollo psicológico y educación. Trastornos del desarrollo y necesidades educativas especiales, v. 3, Alianza Editorial, Madrid, $4^{\mathrm{a}}$ reimpresión, (1 $1^{\text {a }}$ edición, 1990). 2005.

MAURI, T. y VALLS, E. "La enseñanza y el aprendizaje de la geografía, la historia y las ciencias sociales: una perspectiva psicológica”, pp. 509-526 en COLL, C.; PALACIOS, J. y MARCHESI, A. (comp.): Desarrollo psicológico y educación. Psicología de la educación escolar, v. 2., Alianza Editorial, Madrid, $2^{\mathrm{a}}$ reimpresión, (1ª edición, 1990). 2004.

MINISTERIO DE EDUCACIÓN Y CULTURA. Ley Orgánica General del Sistema Educativo, Área de Conocimiento del Medio, Currículo Oficial. Madrid, 1992a.

MINISTERIO DE EDUCACIÓN Y CULTURA. Ley Orgánica General del Sistema Educativo, Área Transversal de Educación Ambiental, Currículo Oficial. Madrid, 1992b.

MINISTERIO DE EDUCACIÓN Y CULTURA. Ley Orgánica General del Sistema Educativo, Área Transversal de Adaptación Curricular, Currículo Oficial. Madrid, 1992c.

MINISTERIO DE EDUCACIÓN Y CIENCIA. Ley Orgánica de Educación, de 3 de agosto, Madrid. 2006. 
OCDE. Escuelas y calidad de la enseñanza. Informe internacional, Paidós y MEC, Madrid, 1990.

O’BRIEN, T. y GUINEY, D. Atención a la diversidad en la enseñanza y el aprendizaje, Alianza Editorial, Madrid, 2003.

OLVERA, F. La investigación del Medio en la Escuela. Penthalón, Madrid. 1987.

RODRÍGUEZ LESTEGÁS, F. "Propuestas para la didáctica del espacio urbano: un enfoque crítico y constructivista”, pp. 91-102 en VV. AA.: El constructivismo en la práctica, Graó, Barcelona, (1 $1^{\mathrm{a}}$ edición, 2000). 2002.

UNIÓN EUROPEA “Competencias clave para el aprendizaje permanente”, Diario Oficial L 394 de 30.12.006. 2006.

VIDAL BOX, C. Guía de recursos pedagógicos de Madrid y sus alrededores. Consejo Superior de Investigaciones Científicas, Madrid. 1976.

Afonso Garcia de la Vega - Doutor em geografia e professor da Universidade Autono[ma de Madrid

Recebido para publicação em junho de 2008 Aceito para publicação em julho de 2008 